\title{
Postmicrosaccadic Enhancement of Slow Eye Movements
}

\author{
Chih-Yang Chen ${ }^{1,2,3}$ and Ziad M. Hafed ${ }^{2,3}$ \\ ${ }^{1}$ Graduate School of Neural and Behavioural Sciences, International Max Planck Research School, Tuebingen, 72074 Germany, ${ }^{2}$ Werner Reichardt Centre \\ for Integrative Neuroscience, Tuebingen, 72076 Germany, and ${ }^{3}$ Animal Physiology, Institute of Neurobiology, University of Tuebingen, Tuebingen, 72076 \\ Germany
}

Active sensation poses unique challenges to sensory systems because moving the sensor necessarily alters the input sensory stream. Sensory input quality is additionally compromised if the sensor moves rapidly, as during rapid eye movements, making the period immediately after the movement critical for recovering reliable sensation. Here, we studied this immediate postmovement interval for the case of microsaccades during fixation, which rapidly jitter the "sensor" exactly when it is being voluntarily stabilized to maintain clear vision. We characterized retinal-image slip in monkeys immediately after microsaccades by analyzing postmovement ocular drifts. We observed enhanced ocular drifts by up to $\sim 28 \%$ relative to premicrosaccade levels, and for up to $\sim 50$ ms after movement end. Moreover, we used a technique to trigger full-field image motion contingent on real-time microsaccade detection, and we used the initial ocular following response to this motion as a proxy for changes in early visual motion processing caused by microsaccades. When the full-field image motion started during microsaccades, ocular following was strongly suppressed, consistent with detrimental retinal effects of the movements. However, when the motion started after microsaccades, there was up to $\sim 73 \%$ increase in ocular following speed, suggesting an enhanced motion sensitivity. These results suggest that the interface between even the smallest possible saccades and "fixation" includes a period of faster than usual image slip, as well as an enhanced responsiveness to image motion, and that both of these phenomena need to be considered when interpreting the pervasive neural and perceptual modulations frequently observed around the time of microsaccades.

\section{Introduction}

Active visual exploration involves frequent transitions between rapid eye movements and fixation. Such transitions also happen even during extended periods of fixation, because tiny saccades, called microsaccades, continue to occur during such periods. Given that saccades and microsaccades alter retinal images, they both modulate neural activity in the visual system. In fact, neural enhancement after saccades/microsaccades has been observed in several areas, including lateral geniculate nucleus, V1, V4, and MT/MST (Bair and O'Keefe, 1998; Leopold and Logothetis, 1998; Martinez-Conde et al., 2000, 2002; Reppas et al., 2002; Ibbotson et al., 2008; Kagan et al., 2008; Rajkai et al., 2008; Bosman et al., 2009; Bremmer et al., 2009; Crowder et al., 2009; Herrington et al., 2009; Cloherty et al., 2010). Although part of this enhancement is due to active extraretinal mechanisms associated with movement generation (Rajkai et al., 2008), a significant component of it also reflects visual reafference after movement end (Martinez-Conde et al., 2000).

The mechanisms of visual reafference after microsaccades are not entirely clear. Specifically, since the eye is never still during

Received Aug. 2, 2012; revised Feb. 5, 2013; accepted Feb. 14, 2013.

Author contributions: C.-Y.C. and Z.M.H. designed research; C.-Y.C. and Z.M.H. performed research; C.-Y.C. and Z.M.H. analyzed data; Z.M.H. wrote the paper.

We were funded by the Werner Reichardt Centre for Integrative Neuroscience, Tuebingen, Germany. We thank Alla Ignashchenkova for helpful comments on an earlier version of this manuscript.

Correspondence should be addressed to Ziad M. Hafed, Werner Reichardt Centre for Integrative Neuroscience, Otfried-Muller Strasse 25,Tuebingen, 72076 Germany. E-mail: ziad.m.hafed@cin.uni-tuebingen.de.

DOI:10.1523/JNEUROSCI.3703-12.2013

Copyright $\odot 2013$ the authors $\quad 0270-6474 / 13 / 335375-12 \$ 15.00 / 0$ fixation (Barlow, 1952), the transition from microsaccades to fixation described above is in fact a more fuzzy transition from fast eye movements to slow, drift-like position displacements. This creates a problem for understanding what contributes to enhanced visual activity after microsaccades. For example, since slow drifts result in retinal-image slip that is within the range of motion sensitivity of the visual system (Verheijen, 1961; Kuang et al., 2012), might it be the case that such image slip is momentarily altered after microsaccades, and thus potentially contributes to the altered visual responses?

Motivated by classic results on large saccades, we have investigated this question by carefully analyzing the patterns of ocular drifts (or, equivalently, retinal-image slip) immediately after microsaccades. We describe two main novel phenomena. First, it is known that large saccades are followed by a period of enhanced ocular drift, sometimes called "glissade" in reference to the smooth change in eye position involved (Weber and Daroff, 1972; Bahill et al., 1978). In the first part of this paper, we demonstrate that glissades remarkably also happen after microsaccades. Second, because glissades alter retinal images exactly when gaze is supposed to be stable, saccades are known to activate a rapid gaze stabilization mechanism immediately after their end. The efficacy of such a mechanism can be observed by imposing retinal image motion on the visual system immediately after saccades and analyzing the ensuing reflexive ocular following eye movement (Kawano and Miles, 1986). In the second part of this paper, we also show that this same mechanism still applies for microsaccades.

In addition to adding to our understanding of the oculomotor and visual mechanisms associated with microsaccades, our work 
more generally highlights an important component of oculomotor activity that studies of postsaccadic perceptual and neural phenomena need to consider: the fact that the transition from even the smallest possible saccades to fixation is not a discrete transition. These studies rightly recognize the importance of understanding postmovement processing for comprehending vision under natural "active" conditions (Rajkai et al., 2008). However, these studies do not always consider the important contributions of postsaccadic drifts to such processing, even though such drifts after large saccades have long been known to exist (Weber and Daroff, 1972; Bahill et al., 1978; Kawano and Miles, 1986).

\section{Materials and Methods}

\section{Animal preparation}

We collected data from two ( $\mathrm{N}$ and $\mathrm{P}$ ) adult, male rhesus monkeys (Macaca mulatta) that were 6 years of age and weighed $6-7 \mathrm{~kg}$. All experimental protocols for the monkeys were in accordance with the guidelines for animal experimentation approved by the Regierungspräsidium (local governing committee) of the city of Tuebingen, Germany.

The monkeys were prepared using standard surgical techniques necessary for behavioral training. Under isoflurane anesthesia and aseptic conditions, we first attached a head-holder to the skull to allow stabilizing head position during the experiments. The head-holder consisted of a titanium implant that was embedded under the skin and attached to the skull using titanium skull screws. We then sutured the skin to cover this implant and allow it to integrate with the bone for several months. In a subsequent surgery, we made a small skin incision on top of the head and attached a metal connector to the previously implanted head-holder. This connector acted as the interface for fixing the head to a standard position in the lab during data collection. In the same surgery, a scleral search coil was implanted in one eye to allow measuring eye movements with high temporal and spatial precision using the magnetic induction technique (Fuchs and Robinson, 1966; Judge et al., 1980).

\section{Behavioral tasks}

Fixation task. To study the characteristics of ocular drifts (or retinalimage slip) before and after microsaccades, we analyzed eye movements from a task in which the monkeys were steadily fixating a small white fixation spot similar to that described by (Hafed et al., 2009), and presented over a uniform gray background (in an otherwise dark room). Each trial lasted for 900-1500 ms, and the monkeys were rewarded for maintaining fixation to within $1-1.5^{\circ}$ from the fixation spot. The spot itself was $\sim 8.5 \times 8.5 \mathrm{~min}$ arc in size, and its luminance was $72 \mathrm{Cd} / \mathrm{m}^{2}$. The background luminance was $21 \mathrm{Cd} / \mathrm{m}^{2}$. The fixation task we used also involved a brief, $\sim 8 \mathrm{~ms}$ luminance transient of the fixation spot (to black and then back to white), and a second brief peripheral white flash $(\sim 50$ $\mathrm{ms}$ ), both occurring at a random time during fixation. These flashes were used to increase microsaccade frequency (Hafed and Clark, 2002; Hafed et al., 2011), for a second ongoing study unrelated to this one, but we are confident that they do not explain the ocular drift results that we present here. In fact, in preliminary analyses, we also tested for premicrosaccadic and postmicrosaccadic alterations in ocular drifts in a second fixation task that did not contain luminance transients, and we found similar results to those presented here.

To compare perimicrosaccadic ocular drifts to those around large saccades, we also measured eye movements in one monkey $(\mathrm{P})$ during a delayed, visually guided saccade task. In this task, the monkey fixated while a peripheral spot was presented (at $5-15^{\circ}$ of eccentricity). Once the fixation spot disappeared, the monkey initiated a visually guided saccade to the peripheral spot.

Ocular following task. To study the sensitivity of early motion processing to retinal-image slip after microsaccades, we presented, in different blocks of trials, a full-field image motion stimulus that was triggered at different times after real-time microsaccade detection. The procedure for this behavioral task was as follows. A static stimulus was first presented to allow the monkeys to fixate the center of the display. This stimulus consisted of a fixation spot presented over the same uniform gray back- ground as described above, along with a large, vertical sine wave grating of 0.25 cycles per degree and $50 \%$ contrast relative to the background luminance. The sine wave grating covered the entire extent of the display, except for a horizontal strip of thickness $22 \mathrm{~min}$ arc and centered vertically on the fixation spot. This strip was of background luminance, and we used it so that the fixation spot was always clearly visible to the monkeys, regardless of the sine wave grating (see Fig. 6A). After the monkeys steadily fixated the central spot (to within $1.5^{\circ}$ ) for at least $500 \mathrm{~ms}$, we enabled a process to detect microsaccades in real-time using eye velocity criteria (see below), and to trigger a pure horizontal motion (rightward or leftward) of the grating contingent on such detection. During the motion, which lasted for $225 \mathrm{~ms}$, the entire grating was shown without the horizontal strip of background luminance. We triggered the motion $0 \mathrm{~ms}, 25 \mathrm{~ms}, 50 \mathrm{~ms}, 75 \mathrm{~ms}, 100 \mathrm{~ms}, 150 \mathrm{~ms}$, or $200 \mathrm{~ms}$ after real-time microsaccade detection, and the motion itself consisted of a horizontal translation of the sine wave grating by $1 / 4$ cycles every $42 \mathrm{~ms}$ (resulting in an effective stimulus speed of $24 \%$ ). If no microsaccade was detected for $500 \mathrm{~ms}$ after enabling the process to detect these movements, we triggered the full-field motion anyway. The fixation spot always disappeared at the same time as motion onset, releasing the monkey from the requirement to fixate, and we analyzed the resulting initial ocular following response to this motion as a proxy for early motion processing (Miles et al., 1986; Masson and Perrinet, 2012; Quaia et al., 2012). We relaxed the fixation constraint of $1.5^{\circ}$ (to $4.5^{\circ}$ ) during stimulus motion to avoid unnecessarily penalizing the monkeys for the ocular following responses that we were interested in measuring. Finally, the initial phase of the sine wave grating was randomly selected on every trial from eight possible equally spaced phases.

We also tested this task on large saccades in one monkey (P), to replicate classic results (Kawano and Miles, 1986). In this variant of the task, the fixation spot initially appeared $10^{\circ}$ to the right or left of the center of the display. Once the monkey fixated this spot, the spot jumped to the center of the display (to its normative position in Fig. 6A), and the monkey initiated a visually guided saccade to follow it (i.e., a $10^{\circ}$ saccade). We removed the spot and triggered full-field image motion of the sine wave grating at different times after real-time detection of the $10^{\circ}$ saccade onset, as described above. Thus, this task variant tested for postsaccadic ocular following responses after large, horizontal $10^{\circ}$ eye movements.

We analyzed 1567 trials from monkey $\mathrm{N}$ and 2818 trials from monkey $\mathrm{P}$ in the microsaccade version of our ocular following task, and we analyzed 1667 more trials from monkey P in the large saccade variant of it.

Experimental control system and real-time microsaccade detection We used a custom-built experimental control system that drove stimulus presentation and ensured monkey behavioral monitoring and reward delivery, as well as implemented real-time microsaccade detection. The core of the system consisted of a real-time signal processor from National Instruments (cRIO-9024), paired to high-speed digital I/O and analogto-digital converter cards. The system ran at $1 \mathrm{kHz}$, and it communicated with our graphics system by sending display update commands using a high-speed universal-data-packet protocol over Ethernet cables. The graphics system in turn consisted of a Mac Pro workstation (Apple) running the Psychophysics Toolbox extension of MATLAB (Brainard, 1997; Pelli, 1997). The graphics system updated display frames at $120 \mathrm{~Hz}$, meaning that our display updated with a maximum possible latency of $\sim 8 \mathrm{~ms}$ ( 1 frame) after microsaccade/saccade detection. All eye movement data and behavioral task events (such as microsaccade detections and display update time stamps) were saved digitally using a dedicated data acquisition system (Multichannel Acquisition Processor; Plexon). We also confirmed (and saved) stimulus update times by measuring display frames using a photodiode aimed at the bottom right corner of the graphics display.

Our system detected microsaccades in real time by obtaining a realtime estimate of radial eye velocity. This estimate used a variant of a previously described algorithm to differentiate the incoming position signals into velocity estimates (Janabi-Sharifi et al., 2000). Briefly, at every time sample, we obtained the slope of a best fitting line to the latest $5 \mathrm{~ms}$ of input eye position data. We then reduced noise in the velocity calculation by obtaining the median of the three latest slope measure- 
ments and using that as our real-time estimate of eye velocity. After estimating eye velocity, we identified microsaccades as the eye movements that exceeded a user-adjustable threshold on this velocity. We manually adjusted the eye-velocity threshold during experiments based on the signal-to-noise ratio in the digitized eye position data. In post hoc analyses, including all analyses presented in this paper, we obtained eye velocities using a more sophisticated "acausal" differentiating filter (oddsymmetric FIR filter with a smoothing $3 \mathrm{~dB}$ cutoff at $54 \mathrm{~Hz}$ ) (Hafed et al., 2009; Lovejoy and Krauzlis, 2010) because in these analyses, we had the luxury of using both past and future eye position samples to estimate derivatives. Such post hoc analyses demonstrated that we could reliably detect microsaccades as small as $3 \mathrm{~min}$ arc in real time (median amplitude: $\sim 12 \mathrm{~min}$ arc), and that the earliest possible display update after true microsaccade onset was $\sim 12 \mathrm{~ms}$ (this included the time for the real-time estimator of velocity to calculate eye speed and the time for the graphics system to start the display update on the next frame). Thus, these post hoc analyses showed that we could reliably start the motion stimulus during tiny microsaccades (whose average duration was $38 \pm 5.9 \mathrm{~ms} \mathrm{SD}$ ), as well as after them, with precise time delays exactly as our experimental design dictated.

For one of our monkeys $(\mathrm{P})$, and in only one analysis in Figure $7 B$, eye movements in the full-field motion task (not the main fixation task above) were collected using a video-based eye tracker (EyeLink 1000;SRResearch) rather than scleral search coils, because the search coil had to be removed from this monkey. The tracker consisted of a high-speed video camera placed under the display and aimed at the monkey's left eye, and its output was directed to the real-time processor exactly as described above. Microsaccades (Otero-Millan et al., 2012) and ocular following responses (Boström and Warzecha, 2009) can both be reliably detected using this system, and we confirmed this with our analyses (see Results) and confirming their similarity to the results with monkey $\mathrm{N}$ using scleral search coils (Figs. 6, 7). We should emphasize, again, that this use of the video-based eye tracker was only restricted to one single analysis (Fig. $7 B$ ) involving smooth ocular following eye movements much faster than fixational drift, and that all other analyses of slow fixational drifts were performed using the more precise scleral search coils. This was important because we found that using a video-based eye tracker like the EyeLink system can cause interpretational ambiguities for slow fixational drifts, as we discuss at length (see Discussion; Fig. 8) and as was also recently found (Kimmel et al., 2012).

\section{Data analysis}

Eye movement detection and classification. Eye movements were sampled at $1 \mathrm{kHz}$. Microsaccades were detected in post hoc analyses using velocity and acceleration thresholds as described by (Krauzlis and Miles, 1996; Hafed et al., 2009). Briefly, for any given microsaccade, we first identified all time samples of the movement in which radial eye velocity exceeded a threshold of $5 \%$. This threshold was chosen to be low enough to detect even the smallest microsaccades, but high enough to exclude eye tracker noise. We then refined the start and end points of the microsaccade by using an acceleration threshold of $250 \%$ s/s. Specifically, after the initial velocity thresholding, we kept marching backward in time (for microsaccade onset) or forward in time (for microsaccade end) below the velocity threshold until absolute eye acceleration was below the acceleration threshold. The latter threshold was again chosen to exclude eye tracker noise from erroneously marking noise samples as parts of microsaccades. In our analyses, we considered as microsaccades all fixational saccades that were $\leq 1^{\circ}$ in radial amplitude. However, in reality, the great majority of these movements were in fact much smaller. Specifically, the overall median microsaccade amplitude in monkey $\mathrm{N}$ was $11 \mathrm{~min}$ arc across all detected microsaccades, and it was 9 min arc in monkey P. These values are consistent with previous reports of microsaccade amplitudes in monkeys (Kagan et al., 2008; Hafed et al., 2009, 2011, 2013; Hafed and Krauzlis, 2010, 2012) and humans (Poletti and Rucci, 2010; Otero-Millan et al., 2012; Hafed, 2013). Moreover, the amplitude distributions of these microsaccades were similar to those published previously, in both monkeys (Kagan et al., 2008; Hafed et al., 2009) and humans (Poletti and Rucci, 2010; Cherici et al., 2012; Otero-Millan et al., 2012; Hafed, 2013), and they showed a skew toward small movement amplitudes. For example, the insets in Figure 2 show such sample amplitude distributions from a subset of our collected microsaccades (those used in the analysis of that figure). As can be seen, the distributions were clearly skewed to small movements, again as previously found (although note that the small sample sizes in these figure insets compared with earlier studies meant that it was even less likely to observe "large microsaccades" in these insets than in previous reports: large microsaccades are normally rare and would thus be even less likely with small sample sizes).

Our use of acceleration criteria to refine microsaccade onset and end times (Krauzlis and Miles, 1996) was critical for the current study because it extended the endpoint of a microsaccade well beyond the endpoint classically identified using methods that only employ eye velocity thresholds. This was of great importance for us because the basic result of our analyses was an enhanced ocular drift velocity after microsaccades (see Results). Thus, we wanted to be as conservative as possible (by extending microsaccade end forward in time) so that we do not attribute velocities that are part of the microsaccade itself (which are necessarily higher than premovement drifts) to our measured postmicrosaccadic drifts. In addition, we added one final step in identifying microsaccade onset and end times. We did so by manually inspecting all detected microsaccades and using all of eye position, eye velocity, and eye acceleration inspection to refine the movement parameters. Our refinement was always toward the conservative side by extending our estimate of movement end forward as much as possible, especially if a microsaccade had a small, but rapid, dynamic overshoot that could contaminate our estimate of postmicrosaccadic drift. For example, the first two sample microsaccades in Figure $1 A$ (from left to right) are movements in which we manually extended their endpoint forward in time beyond the acceleration threshold criterion. We did this to avoid erroneously attributing the small dynamic overshoot present in these movements to postmicrosaccadic drift. Thus, such a conservative approach of using acceleration criteria combined with manual inspection makes us confident that the postmicrosaccadic enhancements of drifts that we report in this paper are not artifacts of erroneously marking the end of a given microsaccade too early, and thus including high velocities from the movement itself in our measurements (see Fig. 1 for example microsaccades and their premovement and postmovement drifts and Fig. 5 for a measurement of eye trajectory after microsaccade end, confirming that the trajectory was not forward in microsaccade direction, as might be expected from a premature marking of microsaccade end).

Time course of premicrosaccadic and postmicrosaccadic drifts. Retinalimage slip is directly related to slow drift velocity during fixation (if the eye drifts in one direction, then the retinal-image "slips" in the opposite direction). Thus, even though earlier studies of premicrosaccadic retinalimage slip have relied on eye position to infer such slip (Engbert and Mergenthaler, 2006), we directly analyzed drift velocity. We measured radial eye velocity during $50 \mathrm{~ms}$ time windows before microsaccade onset or after microsaccade end. To obtain a time course of drift velocity relative to the movement, we slid these time windows backward in time (before microsaccade onset) or forward in time (after microsaccade offset) by $10 \mathrm{~ms}$ steps. To get a statistical estimate of postmicrosaccadic enhancement of drift velocity, we used drift velocity in the $50 \mathrm{~ms}$ period centered at $125 \mathrm{~ms}$ before microsaccade onset as the baseline to which we compared postmicrosaccadic drifts. Moreover, we ensured that any changes in drift velocity (i.e., retinal-image slip) were not contaminated by other microsaccades before or after the movement we were analyzing. We did this by only analyzing premicrosaccadic and postmicrosaccadic drifts for microsaccades that were not preceded by a second movement within $300 \mathrm{~ms}$ and also not followed by a second movement within 300 $\mathrm{ms}$. This ensured that changes in drift velocity associated with a given microsaccade were not artifacts of other nearby eye movements (which necessarily have higher eye velocity than slow drifts), but it meant that the microsaccades analyzed in the current study constituted only a subset of all microsaccades that the monkeys made in our experiments. For some analyses, we extended these time periods even more to 500-600 ms before movement onset (see Results). We used a similar strategy when analyzing large saccade data.

In some of our analyses, we reported relative measures of drift velocity after microsaccades. That is, we normalized eye velocity relative to the 
baseline velocity measurement made at a time point centered on $125 \mathrm{~ms}$ before movement onset. This approach ensured that our results were not dependent on our particular choice for estimating eye velocity. For example, depending on the amount of smoothing performed during eye position differentiation in different algorithms, the absolute measure of eye velocity during slow drifts can be variable (Cherici et al., 2012). However, by using relative measures and a single algorithm for all analysis time windows, it was still possible to reliably identify relative changes in postmicrosaccadic drift as a function of time after microsaccades. Having said that, in Figures 1 and 2, we also analyzed absolute measures of eye velocity without any normalization, and we confirmed that the basic results we are reporting were unaltered by such normalization procedure.

To further confirm our results on postmicrosaccadic changes in drift, we also assessed such drift using a second method completely independent of a specific algorithm for eye velocity estimation. Specifically, and like previous studies of premicrosaccadic retinal-image slip that have used eye position as a proxy for this slip (Engbert and Mergenthaler, 2006), we analyzed premicrosaccadic and postmicrosaccadic image slip by directly analyzing eye positions. We used the same "box-counting" procedure used earlier (Engbert and Mergenthaler, 2006), but we extended it to measure eye drift after microsaccades, not just before them. This box-counting procedure may be thought of as "path integration" of eye trajectory. Briefly, within a given $50 \mathrm{~ms}$ time period relative to microsaccade onset or end, the $2 \mathrm{D}$ locations of eye position in this period are spatially binned $\left(0.01^{\circ}\right.$ bin width), and the count of spatial bins (boxcount) covered by the eye trajectory during this $50 \mathrm{~ms}$ time period provides an estimate of how much "space" the eye has traversed along its "path." If the eye drifts faster than usual, then the box-count is increased, and vice versa if the eye drifts slower than usual. We compared the results of this box-counting procedure to our results with direct measures of retinal-image slip (through measures of ocular drift velocity), to confirm our interpretation of postmicrosaccadic enhancements using two different techniques.

Finally, we used eye position to also investigate the spatial trajectory of ocular drift immediately after microsaccades. We simply measured eye position in every millisecond after microsaccade end, and we averaged all such measurements for all detected microsaccades, but only after rotating all microsaccades (and the postmovement measurements) to align all data for a "canonical" microsaccade ending direction. Thus, the measured postmicrosaccadic drift positions were calculated relative to the direction of the final quarter of the antecedent microsaccade. This averaging technique allowed us to conclude whether postmicrosaccadic ocular drift (and thus retinal-image slip) was systematically related to the trajectory of the antecedent movement or not. If it is, then this averaging technique should reveal a consistent trajectory. Otherwise, it should not. We then repeated the same procedure for the drift trajectory immediately before microsaccade onset. In this case, we rotated all measurements to measure eye position relative to the direction of the initial quarter of microsaccade trajectory.

Time course of ocular following responses after microsaccades. To test for a possible consequence of postmicrosaccadic drift on the visual system, we used our full-field image motion task to artificially simulate full-field retinal-image slip at different times after microsaccades. Full-field image motion is known to drive an ultrashort-latency ocular following reflex that attempts to stabilize this motion (Miles et al., 1986; Gellman et al., 1990; Masson and Perrinet, 2012). The initial component of this ocular following response is an "open-loop" response that is not influenced by visual feedback caused by ongoing eye movements, and thus acts as a proxy for the very initial motion processing of the full-field (retinal) image motion (Masson and Perrinet, 2012; Quaia et al., 2012). Thus, we analyzed only the initial ocular following response after full-field motion onset. For every trial, we measured the peak eye velocity during the time period between 80 and $100 \mathrm{~ms}$ after motion onset. To measure the time course of modulations in this response after microsaccades, we manually inspected all microsaccades as described above, and we binned trials based on when the motion started after microsaccade offset. We then plotted the magnitude of the ocular following response (peak eye velocity in the period between 80 and $100 \mathrm{~ms}$ after motion onset) as a function of the time of motion onset relative to microsaccade offset.

\section{Results}

\section{Ocular drift (or retinal-image slip) was constant before microsaccades but enhanced immediately afterward}

We analyzed 2314 microsaccades from two male rhesus macaques fixating a small fixation spot. For each of these microsaccades, whose overall median amplitude was $8.24 \mathrm{~min}$ arc for monkey $\mathrm{N}$ and $4.54 \mathrm{~min}$ arc for monkey $\mathrm{P}$, we analyzed slow ocular drift velocity before and after the movements. This allowed us to infer the pattern of retinal-image slip around the time of even the smallest of microsaccades. We carefully measured eye movements in both monkeys using the magnetic induction technique (Fuchs and Robinson, 1966; Judge et al., 1980), which was critical for interpreting our results. Not only does this technique have unparalleled spatial and temporal resolution, but it also has the added advantage of not exhibiting postmovement ringing that is frequently observed with video-based measurements of eye position; these video-based measurements are affected by factors such as ocular lens distortions during rapid eye movements, which become particularly prominent at the ends of the movements (Deubel and Bridgeman, 1995), and which would otherwise potentially mask the postmovement effects that we were studying.

Postmicrosaccadic drift velocity was consistently enhanced relative to premovement levels, even for the tiniest eye movements. Figure $1 A$ shows three sample microsaccades of different sizes and directions, for which a clear postmovement ocular drift was observed in both eye position and eye velocity traces. The figure plots horizontal and vertical eye position (top row) as well as radial eye velocity (middle row) and acceleration (bottom row) before, during, and after these tiny saccadic eye movements. The eye position, velocity, and acceleration samples marked in green in the figure represent the microsaccadic component of the eye movement, and the other samples show premovement or postmovement drift. As can be seen, for all of these sample microsaccades, there was a clear enhancement of ocular drift velocity immediately after microsaccades relative to before the rapid eye movements (highlighted with a black diagonal arrow for each microsaccade), and this enhancement was also evident in the eye position traces as well. In fact, the eye position traces revealed that such faster postmicrosaccadic drifts could be characterized as so-called "glissadic overshoots" (in which the eye drifts backward immediately after movement end) commonly observed after much larger saccades (Bahill et al., 1978). An example of such a glissadic overshoot after large saccades from our own dataset is illustrated in Figure $1 B$, in which we document a postmovement drift after a large $15^{\circ}$ visually guided saccade generated by monkey P. Thus, our analysis of the sample microsaccades in Figure $1 A$ shows that, just like for their much larger counterparts (Fig. $1 B)$, ocular drift, and necessarily retinal-image slip, was markedly enhanced (i.e., faster) after these tiny eye movements, despite the fact that these movements (like the first two in Fig. 1A) could be smaller than even the strictest definitions for microsaccades (Collewijn and Kowler, 2008; Hafed et al., 2009; Hafed and Krauzlis, 2012).

Postmicrosaccadic enhancement of ocular drift velocity was consistently observed in each of the two monkeys. For every detected microsaccade in one sample session from each of our monkeys, we analyzed premicrosaccadic and postmicrosaccadic ocular drift velocity as we did for the three sample movements of Figure $1 A$ above. We specifically measured radial eye velocity 
A

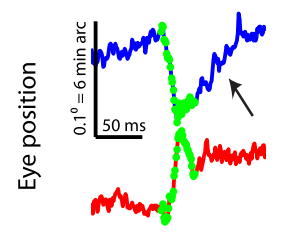

Microsaccades
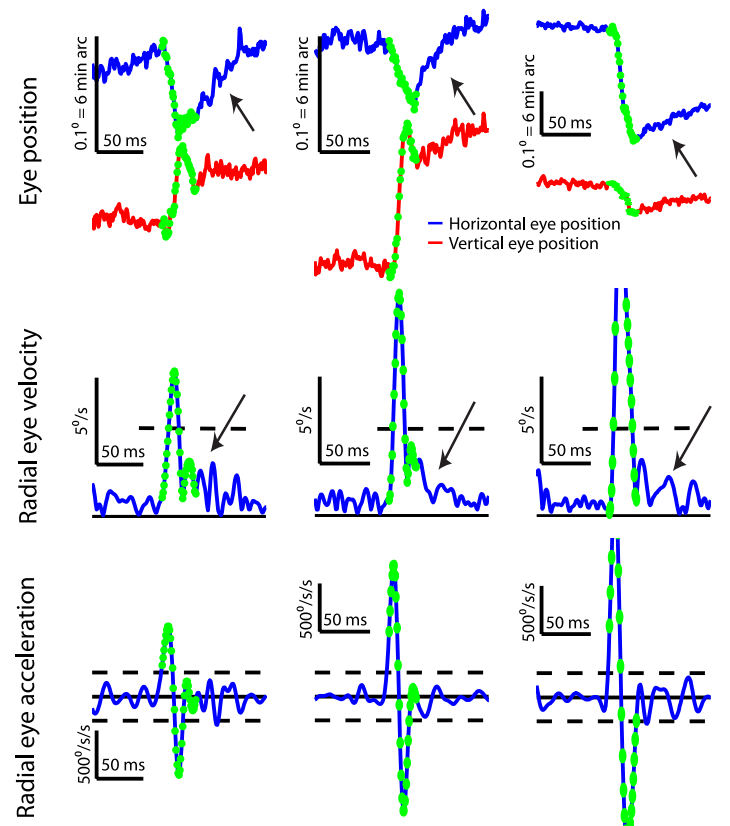

Time

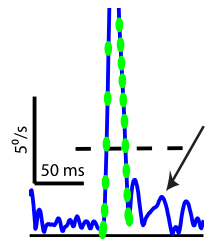

B 15-Deg saccade
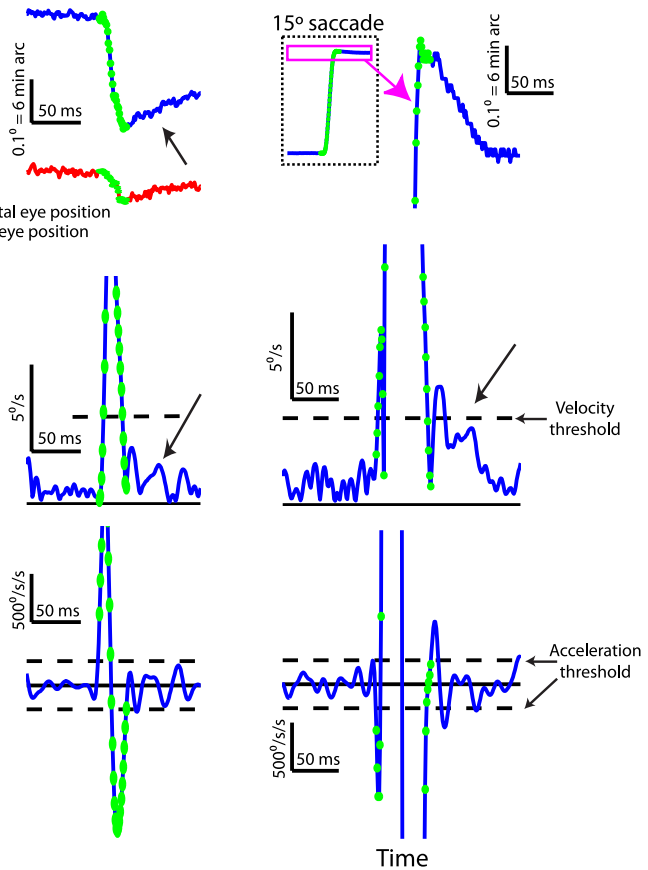

Figure 1. Postmicrosaccadic enhancement of slow eye movements. $\boldsymbol{A}$, Three example microsaccades of increasing size (from left to right) and different directions showing differences between premovement and postmovement drift. The top row shows horizontal (blue) and vertical (red) eye position, the middle row shows radial eye velocity, and the bottom row shows radial eye acceleration. The dashed horizontal lines in the middle and bottom rows indicate the velocity (middle) and acceleration (bottom) thresholds we used to mark microsaccade onset and end (see Materials and Methods), and the green samples are those that were flagged as part of a microsaccade. Note that for the first two microsaccades, we manually extended microsaccade end forward in time to ensure that the small dynamic overshoot in vertical eye position did not artifactually bias our estimates of postmicrosaccadic drifts (see Materials and Methods). The black diagonal arrows highlight a period of clear enhancement of postmicrosaccadic drift (compare position and velocity traces after microsaccades to a similar period before them). $\boldsymbol{B}$, An illustration of postsaccadic drift (Weber and Daroff, 1972; Bahill et al., 1978) for a large, $15^{\circ}$ saccade. A conceptually identical postmovement drift was observed as in the case of the microsaccades in $\boldsymbol{A}$, albeit with a larger overall magnitude and duration. In all the eye-position plots, upward deflections in the traces denote rightward and upward changes in eye position, respectively.

during the first $50 \mathrm{~ms}$ after microsaccade end (i.e., during a $50 \mathrm{~ms}$ time window centered on $25 \mathrm{~ms}$ after movement end) and compared it to radial eye velocity during a $50 \mathrm{~ms}$ time window centered around $125 \mathrm{~ms}$ before microsaccade onset. Across the analyzed population of microsaccades in these two sample sessions, there was a robust enhancement in ocular drift velocity immediately after microsaccades relative to the premovement baseline (Fig. $2 A, C)(p<0.0001$ for monkey $\mathrm{N}$ and $p<0.0001$ for monkey P; paired $t$ test). Specifically, the average drift velocity in monkey $\mathrm{N}$ was $\sim 1 \%$ s before microsaccades but $\sim 1.28 \% \mathrm{sim}-$ mediately after the movements ( $\sim 28 \%$ enhancement), and it was $\sim 0.74 \%$ before microsaccades but $\sim 0.91 \%$ after them for monkey $\mathrm{P}$ ( $\sim 23 \%$ enhancement). Moreover, this effect was specific to the postmovement period, because a similar analysis but now measuring radial eye velocity during two premicrosaccade intervals showed no significant enhancement or reduction (Fig. $2 B, D)(p=0.1$ for monkey $\mathrm{N}$ and $p=0.6$ for monkey P; paired $t$ test). Thus, immediately after microsaccade end, ocular drift (and thus retinal-image slip) was consistently enhanced in both monkeys, and this effect was linked to the antecedent eye movement because no change in ocular drift was apparent before the movement.

The above analyses compared ocular drift velocity before and after microsaccades to demonstrate a possible postmicrosaccadic enhancement effect. However, it may be argued that the enhance- ment we observed in Figures 1 and 2 after microsaccades was simply an artifact of reduced "retinal-image slip" before the movements, as was recently suggested to happen (Engbert and Mergenthaler, 2006). In other words, it could be argued that postmicrosaccadic ocular drift is not enhanced, but it is simply the premicrosaccadic drift that is suppressed instead. To rule this possibility out, we analyzed the full-time course of premicrosaccadic and postmicrosaccadic ocular drifts, rather than just during two temporal windows before and after the movement as we did above in Figure 2. The results of this analysis are shown in Figure $3 A, B$ for each monkey individually (black curves). To obtain this figure, we measured radial eye velocity (or the radial velocity of retinalimage slip) during $50 \mathrm{~ms}$ time windows that were centered at $10 \mathrm{~ms}$ time steps relative to either microsaccade onset or microsaccade end. To facilitate comparisons of eye velocity at different time windows, we normalized all measurements to the radial eye velocity during the $50 \mathrm{~ms}$ window centered on $-125 \mathrm{~ms}$ relative to microsaccade onset. As can be seen, we saw no evidence for a reduction in drift velocity before microsaccade onset and for up to $200 \mathrm{~ms}$ before such onset. Instead, drift velocity remained relatively constant, and it was strongly enhanced for a short interval after the end of the microsaccades. For example, monkey $\mathrm{N}$ showed a $25.7 \pm$ $1.1 \%$ SEM enhancement in ocular drift velocity during the first $50 \mathrm{~ms}$ after microsaccade end, and monkey $\mathrm{P}$ showed a $17.6 \pm 0.7 \%$ SEM enhancement in the same time window. We also noticed that both monkeys showed a modest enhancement immediately before microsaccade onset, but this enhancement was much smaller than that observed after microsaccade end. Moreover, these effects did not depend on microsaccade size, because they persisted for movements that were only smaller than the median amplitude in our population (green curves) as well as movements that were only larger than the median amplitude (red curves). We also analyzed large $5-15^{\circ}$ saccades from monkey $\mathrm{P}$ (Fig. 3B, blue curves in the insets), and we found that our microsaccadic results were conceptually identical to those of classic "glissades" after large saccades (consistent with Fig. 1B), although they were smaller and more short-lived. Thus, the postmicrosaccadic enhancement in ocular drift velocity that we observed above (Figs. 1,2) was directly linked to the microsaccade, and it was not an artifact of premovement reductions in retinal-image slip, as was recently suggested (Engbert and Mergenthaler, 2006).

We further analyzed the time course of premicrosaccadic and postmicrosaccadic ocular drift, this time by analyzing eye position using the exact same path integration (or box-counting) algorithm as that used in (Engbert and Mergenthaler, 2006) (see Materials and Methods). To further confirm that retinal-image slip was relatively unaltered before microsaccades and only increased after the movements, we extended for this analysis our time intervals for measuring premovement and postmovement 

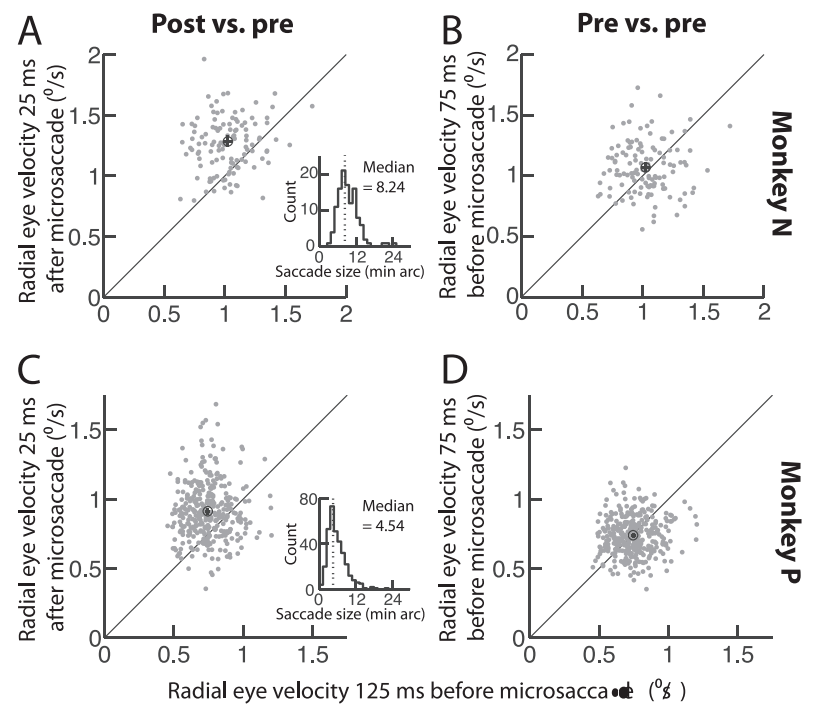

Figure 2. Postmicrosaccadic enhancement occurred in both monkeys. $A, C$, Comparison of radial eye velocity during a $50 \mathrm{~ms}$ time window immediately after microsaccade end (i.e., centered on $25 \mathrm{~ms}$ after the microsaccade) ( $y$-axis) to radial eye velocity during a $50 \mathrm{~ms}$ time window well before microsaccade onset (i.e., centered on -125 ms relative to movement onset) ( $x$-axis). In each panel, the individual symbols correspond to individual microsaccades in monkey $N(\boldsymbol{A})$ or monkey $P(\boldsymbol{C})$. The black hollow circle in each panel shows mean values. Error bars around this circle (horizontal and vertical), when visible, indicate $95 \%$ confidence intervals. As can be seen, there was a robust enhancement in drift velocity after microsaccades relative to before them. The inset in each panel shows the amplitude distribution of the microsaccades in this figure (note that such distribution was skewed to small microsaccades, consistent with previous reports, but that this skew is particularly more obvious here because of the small sample size: large microsaccades are normally already rare, so they are even less likely to be observed with such a small sample size). $\boldsymbol{B}, \boldsymbol{D}$, Similar analyses from the same session in each monkey, but now comparing eye velocity during two time windows that were both before microsaccade onset. No enhancement or reduction occurred.

drift. Specifically, in Figure $3 A$ and $B$ above, we only measured drift velocity from $200 \mathrm{~ms}$ before microsaccade onset to $200 \mathrm{~ms}$ after microsaccade end. However, for the present analysis, we picked only the microsaccades that were not preceded by other microsaccades by up to $500-600 \mathrm{~ms}$. This allowed us to analyze box-counts during longer time intervals spanning from 400 to $500 \mathrm{~ms}$ before microsaccade onset. For both monkeys, the boxcount obtained during $50 \mathrm{~ms}$ ocular drift intervals was significantly increased in the immediate postmovement interval relative to premicrosaccade levels, consistent with our previous observations above, but it was again virtually unaltered for the entire 400-500 ms before microsaccade onset (Fig. 4A, C). Thus, contrary to (Engbert and Mergenthaler, 2006), we saw no evidence that retinal-image slip is reduced before microsaccades. Instead, the most obvious observation in our data was that postmovement slip was significantly enhanced. We further confirmed this by measuring ocular drift velocity on the same movements analyzed in Figure 4, $A$ and $C$, and having the same longer premovement analysis intervals (Fig. $4 B, D$ ). Both eye position and eye velocity analyses revealed relatively stable drift before microsaccades and a strong, but short-lived postmicrosaccadic enhancement.

Postmicrosaccadic ocular drift was related to the direction of the antecedent microsaccade

Since our analyses above suggested that postmicrosaccadic enhancement of ocular drift velocity was directly related to the immediate generation of an antecedent microsaccade, we next asked whether the trajectory of the eye after the movement was in any

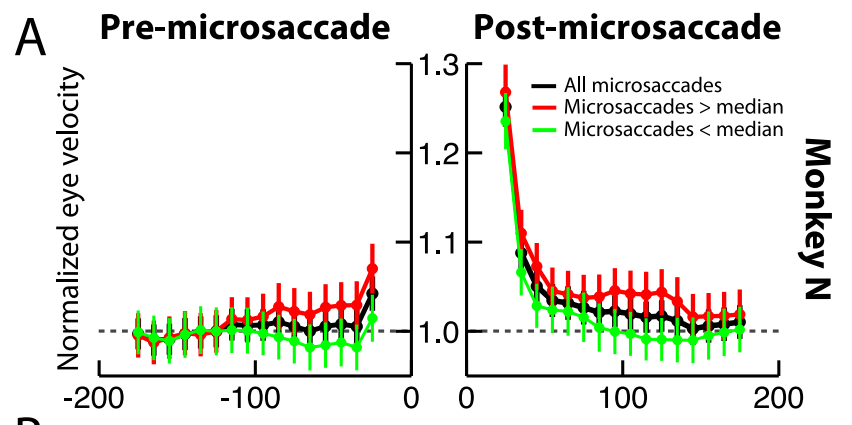

B

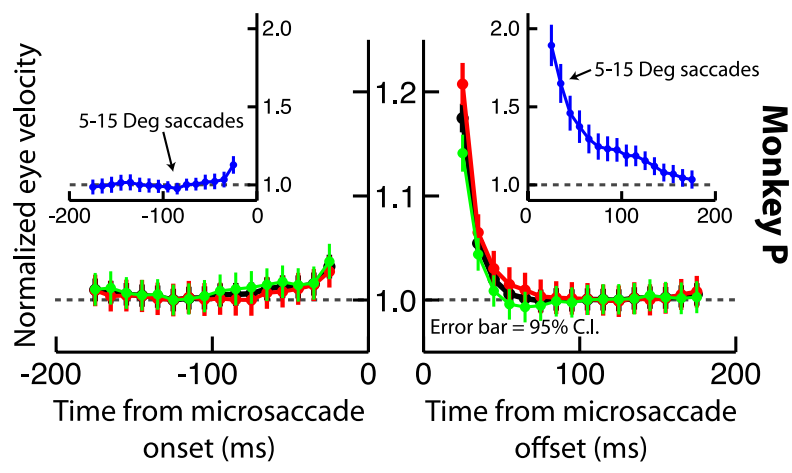

Figure 3. $\quad A, B$, Perimicrosaccadic drift velocities were relatively constant long before microsaccades, but enhanced after them. In each panel, we plotted in black radial eye velocity for each monkey as a function of time from microsaccade onset (left subgraph) or microsaccade end (right subgraph). All eye velocities were first normalized to the velocity measured during a 50 ms time window centered on $-125 \mathrm{~ms}$ from microsaccade onset. We then measured eye velocities in similar $50 \mathrm{~ms}$ time windows that were translated in time. Drift velocity was relatively constant before microsaccade onset, but it increased immediately after the movements. The red curves show the same analysis but only for microsaccades larger than the median amplitude, and the green curves show it for microsaccades smaller than the median amplitude. The blue curves in the inset for monkey P show data from $\sim 450$ large $\left(5-15^{\circ}\right)$ saccades made by this monkey. These additional curves demonstrate that the basic conceptual results did not depend on movement amplitude. Error bars in all parts indicate $95 \%$ confidence intervals.

way related to the direction of the microsaccade that was just completed (as we observed in the sample microsaccades of Fig. $1 A)$. In other words, is postmicrosaccadic drift corrective in nature (i.e., does the eye drift back opposite the microsaccade direction?) or does it exhibit momentum (i.e., does the eye drift forward in the same direction as a microsaccade?) or is it completely unrelated to the antecedent movement? To answer this question, we measured for every microsaccade the position of the eye in every millisecond after movement end. Because our monkeys made microsaccades in all directions (Fig. 1), and to combine microsaccades of different directions in the same summary analysis, we first applied a coordinate transformation (rotation) such that all microsaccades (and subsequent drifts) were aligned to a "canonical" direction based on the direction of the final trajectory of the microsaccades (see Materials and Methods). Our motivation for this analysis was as follows: if postmicrosaccadic drift is completely unrelated to the antecedent microsaccade trajectory, then averaging across all movements should not result in any systematic pattern of postmovement drift. However, if the postmovement drift is consistently related to the direction of the previous microsaccade, then averaging should reveal a consistent path. Figure 5, $A$ and $C$, shows the result of this analysis for each monkey. In this figure, we plotted the average horizontal and vertical eye position of the monkey relative to the ending position of a microsaccade (indicated schematically as the origin of the figure), and we rotated all axes such that positive horizontal axes 

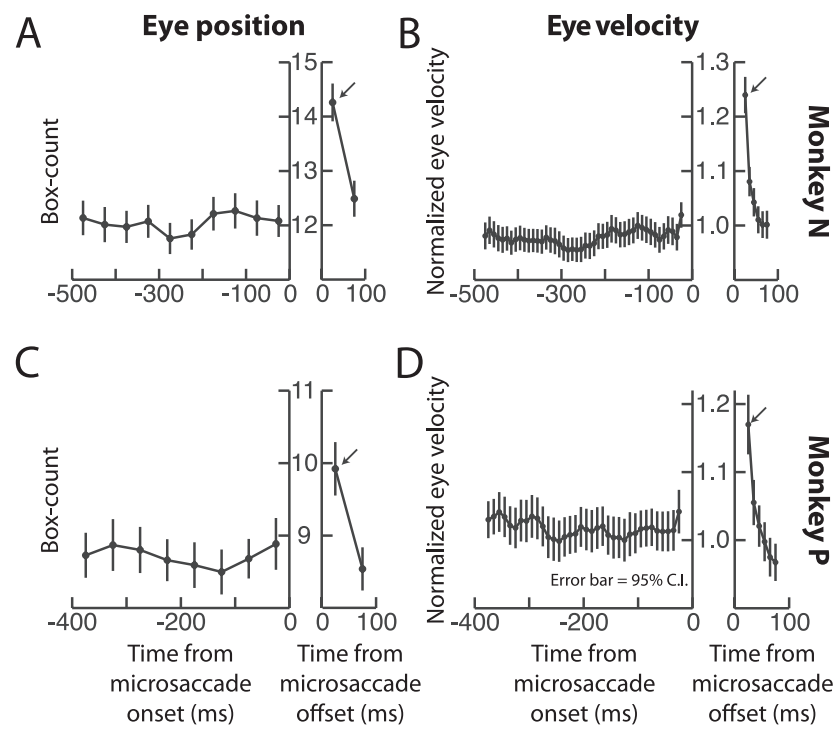

Figure 4. Postmicrosaccadic enhancement was also observed using eye position estimates of ocular drift. $A, C$, For each monkey, we implemented the box counting path integration algorithm by Engbert and Mergenthaler (2006). However, in our case, we measured box counts both after microsaccade end as well as before microsaccade onset, instead of just in the latter interval. Box counts of eye position drift were constant before movement onset, unlike in the data of Engbert and Mergenthaler (2006). However, consistent with our earlier analyses above, box counts were larger after microsaccade end. $\boldsymbol{B}, \boldsymbol{D}$, We further tested the same eye movements in $\boldsymbol{A}$ and $\boldsymbol{C}$, but this time by analyzing eye velocities similar to our procedure in Figure 3 . Consistent with $\boldsymbol{A}$ and $\boldsymbol{C}$, eye velocity for these same movements was constant for up to $500 \mathrm{~ms}$ before microsaccade onset, and it was enhanced immediately after movement end. Error bars in all plots indicate $95 \%$ confidence intervals. Note that monkey $P$ made frequent microsaccades, so it was virtually impossible to find fixation intervals in which a single microsaccade was not preceded by an earlier movement for a full $500 \mathrm{~ms}$. Thus, for this monkey, we restricted the premicrosaccadic interval to $400 \mathrm{~ms}$, to avoid contamination of ocular drift measurements by other previous microsaccades.

in the figure were drifts in the same direction as the final microsaccade trajectory and positive vertical axes were drifts orthogonal (and counterclockwise) to the direction of the antecedent microsaccade. As can be seen, in both monkeys, postmicrosaccadic drift had a corrective component to it. Specifically, in both monkeys, the eye consistently drifted backward directly opposite the antecedent microsaccade's ending trajectory. Note how the enhancement in postmicrosaccadic drift velocity is also obvious in this figure, evidenced by the larger displacement of the average eye position in every millisecond of time during the initial period after the movement end. Also note that this analysis confirms that we did not prematurely mark microsaccade end, and thus erroneously treat saccadic components of eye velocity as postmicrosaccadic drift, because in this case Figure 5, $A$ and $C$, should reveal a trajectory along the path of the yet-to-end microsaccade rather than opposite it as we observed. Thus, in both monkeys, not only was postmicrosaccadic drift faster than normal, but it also exhibited a corrective component relative to the antecedent movement direction, as is the case with so-called glissadic overshoots in much larger saccades (Bahill et al., 1978; Fig. 1B). Such corrective component was also seen in the sample microsaccades of Figure $1 A$. For comparison, we also plotted in Figure 5, $B$ and $D$, results of the same analysis but now for premicrosaccadic drift instead. In this case, we plotted premovement horizontal and vertical average eye position relative to the starting point of a microsaccade, and all microsaccades were again rotated such that the horizontal axis reflected positions along the initial direction of the upcoming movement and the vertical axis reflected positions or-
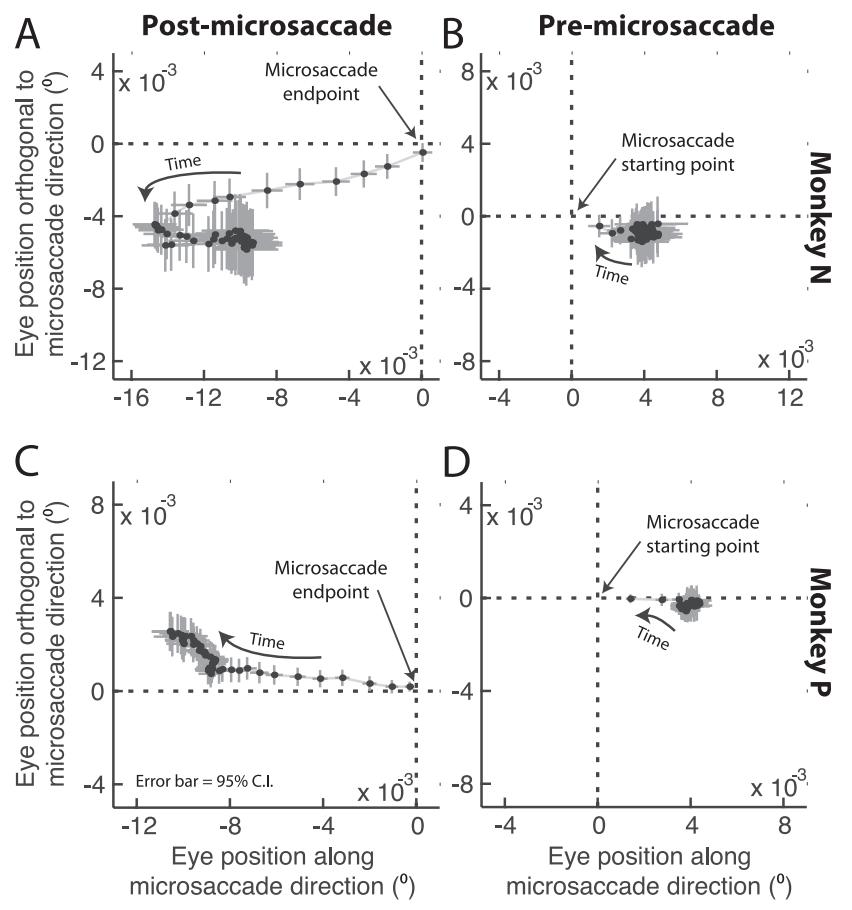

Figure 5. Postmicrosaccadic drift trajectory was related to the antecedent microsaccade direction. $A, C$, We plotted average eye position in every millisecond following microsaccade end. Each panel plots the first $50 \mathrm{~ms}$ of eye drift after microsaccades. Each symbol shows the average $\pm 95 \%$ confidence intervals, and the first point after microsaccade end is the point closest to the origin in the figure. The progression of time after a microsaccade is indicated graphically by an arrow. As mentioned in the text, before averaging such eye positions, we first re-aligned all microsaccades according to the direction of the final quarter of microsaccade trajectory, such that rightward horizontal displacements in the figure now correspond to drifts along the same direction as the antecedent microsaccade and upward vertical displacements in the figure correspond to drifts orthogonal to the direction of the antecedent microsaccade. For both monkeys, postmicrosaccadic drift was consistently opposite the final direction of the antecedent eye movement. $\boldsymbol{B}, \boldsymbol{D}$, Similar analyses but now for eye positions immediately before microsaccade onset. This time, we aligned movements according to the initial microsaccade trajectory such that rightward displacements in the figure are displacements along the upcoming microsaccade direction. Notice how premicrosaccadic drift was slow (strong clustering of the points together) compared with postmicrosaccadic drift, and that it did not exhibit a consistent trajectory.

thogonal to its direction. As can be seen, no consistent pattern of eye position, or enhancement, was seen before microsaccades. Thus, the analyses in Figure 5 combined confirm that retinalimage slip is enhanced immediately after microsaccades, and they also show that the direction of such slip has a corrective component to the slip caused by the antecedent eye movements. Such corrective component was also recently observed in humans immediately after microsaccades (Cherici et al., 2012).

\section{Ocular following responses to full-field image motion were also enhanced after microsaccades}

One possible hypothesis about the postmicrosaccadic enhancement described above is that the faster retinal-image slip it gives rise to can activate visual circuitry in the retina and early visual system with full-field image motion caused by the drifting eye movement. This full-field image motion can in turn galvanize a "field-holding reflex" mediated by activity in the early visual system in response to such image motion, as is hypothesized to happen for much larger saccades (Kawano and Miles, 1986). If this is the case, then artificially altering the full-field image motion impinging on the retina immediately after microsaccades, to simulate larger than usual retinal-image slip, should tap into the 
same putative "microsaccade-activated" field-holding reflex. This would in turn become reflected in subsequent reflexive eye movements that attempt to stabilize the retinal image of the now artificially generated full-field motion. We tested this hypothesis explicitly by designing a task that probed low-level motion sensitivity in the visual system immediately after microsaccades. The task relied on a stimulus like that shown in Figure 6A, consisting of a low-frequency, full-field sine wave grating (Sheliga et al., 2008; Quaia et al., 2012). Whenever we detected a microsaccade during fixation, we triggered a horizontal motion of this sine wave grating either during the movement itself or at different times after it (see Materials and Methods). Such horizontal full-field image motion is known to drive an ultrashort-latency ocular following response (Miles et al., 1986; Gellman et al., 1990; Matsuura et al., 2008; Sheliga et al., 2008; Masson and Perrinet, 2012), which is thought to reflect motion responses in the early visual system (Quaia et al., 2012). Our goal was to find out whether such a response can be modulated by antecedent microsaccades.

Full-field horizontal image motion resulted in a short-latency ocular following response without any microsaccades, as is expected from prior work (Miles et al., 1986). We first confirmed the presence of ocular following responses in our monkeys by analyzing the eye velocity in response to full-field image motion whenever this motion started after at least $213 \mathrm{~ms}$ from the end of any previous microsaccades. Figure $6 B$ shows the average eye velocity for all trials in which this was the case from one of our monkeys $(\mathrm{N})$. As can be seen, radial eye velocity was stable for several tens of milliseconds before the full-field motion onset. However, starting $\sim 80 \mathrm{~ms}$ after motion onset, there was an initial increase in eye velocity in response to the motion (arrow) (note that there was an even earlier bump in eye velocity at $\sim 50 \mathrm{~ms}$, but we elected to analyze the slightly later increase to avoid any possible contamination by eye tracker noise). This initial increase represents a rapid image-stabilizing reflex by the visual system (Miles et al., 1986), and it reflects pooling of early motion signals to drive the eye in the same direction as the motion (Masson and Perrinet, 2012), and thus effectively reduce the motion's slip on the retina.

If microsaccades do indeed cause faster than usual retinalimage slip after their end (Figs. 1-5), then one might expect that these eye movements would activate a field-holding reflex to quickly stabilize such slip, just like larger saccades do (Kawano and Miles, 1986). We thus asked whether the basic ocular following reflex in Figure $6 B$ was in any way modulated by microsaccades. To do this, we plotted radial eye velocity in the same manner as in Figure 6B, but now for only the trials in which the motion of the sine wave grating started within a specific time interval relative to microsaccade end. An example of one such interval is shown in Figure $6 C$, in which we only considered all the trials with the motion starting immediately upon microsaccade detection (i.e., the motion started during a microsaccade) (black curve). For comparison, we show in the same figure the ocular velocity after motion onset when no microsaccades occurred (gray curve; which is an identical copy of the curve in Fig. $6 \mathrm{~B}$ but placed here to facilitate comparison). As can be seen, whenever the motion onset started during microsaccades, the subsequent ocular following responses were dramatically suppressed, and for a prolonged period of time. This is consistent with the detrimental visual effects of rapid eye movements, and the possible involvement of active suppressive mechanisms associated with these movements (Hafed and Krauzlis, 2010). However, when we repeated the same analysis but now for the trials in which the motion started within $\sim 50 \mathrm{~ms}$ after microsaccade end, we found a large, almost twofold enhancement in ocular following response (Fig. 6D). Thus, full-field retinal-image motion that was presented immediately after microsaccades resulted in a much-enhanced magnitude of the image-stabilizing reflex by the oculomotor system, compared with identical full-field retinal-image motion that was presented without any antecedent microsaccades.

Similar to the postmicrosaccadic enhancement of ocular drift velocity we observed earlier (Figs. 1-4), the postmicrosaccadic enhancement of ocular following responses to full-field image motion was also time locked to movement end, and it was consistent across both monkeys. For each monkey, we analyzed the initial ocular following response (peak eye velocity in the period between 80 and $100 \mathrm{~ms}$ after motion onset; see Materials and Methods), and we plotted the magnitude of this response as a function of when the full-field motion started relative to microsaccade end (Fig. $7 A, B$ ). Consistent with the sample data in Fig- 


\section{A Peri-microsaccadic OFR}
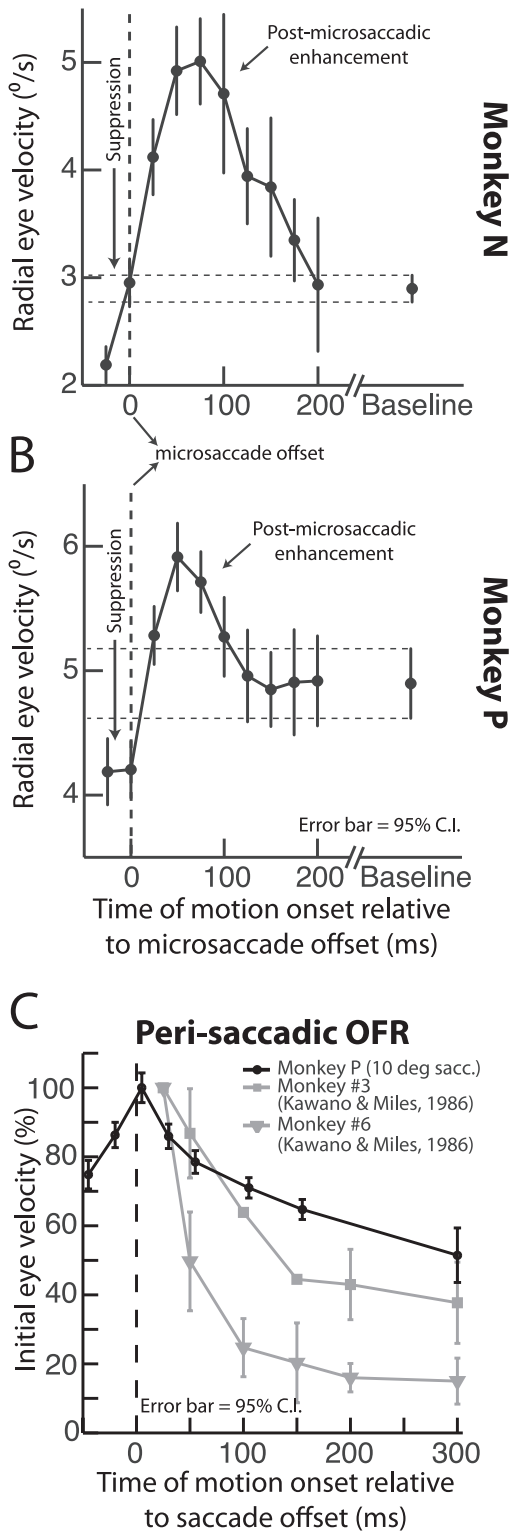

Figure 7. Time course of ocular following response magnitude relative to microsaccades. $A, B$, We plotted the magnitude of the initial ocularfollowing response (see measurement box in Fig. $6 B-D$ and Materials and Methods) as a function of the time of full-field motion onset relative to microsaccade end. Consistent with the sample data of Figure 6, motion onset during microsaccades was associated with a strong suppression in the ocular following response (notice the response for negative time values on the $x$-axis). However, motion onset immediately in the wake of microsaccades was associated with strong enhancement in sensitivity to the full-field motion stimulus. This enhancement was then followed by a gradual return to baseline ocular following performance. C, Data from monkey $\mathrm{P}$ (black) showing ocular following response magnitude after $10^{\circ}$ saccades instead of microsaccades. The data are plotted in a formatidentical to that used classically (Kawano and Miles, 1986), to facilitate comparison to classic results. We also replicated (with permission from the publisher) representative data from two monkeys reported by Kawano and Miles (1986) to demonstrate the consistency of our results. Similar to Figure 3, large saccades exhibited a conceptually identical result to microsaccades, albeit stronger and longer lived. Note that idiosyncratic differences in time course for monkey P relative to the monkeys of Kawano and Miles (1986) likely reflect the different motion stimuli used, as well as the differences in algorithms used for detecting exact saccade end. Error bars in all panels indicate $95 \%$ confidence intervals.

ure $6 B-D$, image motion that started during microsaccades was strongly suppressed in terms of its influence on subsequent ocular following (compare the response to that without any nearby antecedent saccades; rightmost Baseline point in each panel).
However, whenever the image motion started within $\sim 50-75 \mathrm{~ms}$ after microsaccade end, there was a large and significant enhancement in eye velocity compared with Baseline. This enhancement and its time course were similar to the enhancement known to happen after much larger saccades, although it was smaller in absolute magnitude, peaking at $\sim 73 \%$ as opposed to $\sim 100-$ $400 \%$ for much larger saccades according to the classic literature (Kawano and Miles, 1986). We also confirmed this observation by testing monkey P using a "large-saccade" variant of the same full-field image motion task (see Materials and Methods). We found that this monkey exhibited similar postsaccadic enhancement as observed classically (Kawano and Miles, 1986), and that the peak enhancement was bigger than for microsaccades. In particular, Figure 7C plots this monkey's large-saccade ocular following responses in a manner identical to that shown in (Kawano and Miles, 1986, their Fig. 2). For comparison, we also included in this figure data from two of the monkeys used in that earlier classic study. As can be seen, our monkey showed a $\sim 100 \%$ peak magnitude postsaccadic enhancement of ocular following responses relative to $\sim 300 \mathrm{~ms}$ after saccades, which is similar to some monkeys in the earlier classic study (e.g., Monkey \#3) (Kawano and Miles, 1986), and which is larger than the postmicrosaccadic enhancement. Thus, not only did we observe an enhancement in postmicrosaccadic ocular drift (Figs. 1-5), but we also found postmicrosaccadic enhancement in imagestabilizing ocular responses (Figs. 6,7), which are presumed to be helpful for quickly counteracting such postmicrosaccadic drift under normal conditions. Moreover, these responses were conceptually similar to those after much larger saccades.

\section{Summary}

In summary, our results above demonstrate two main findings. First, the transition between even the tiniest of saccades and subsequent fixation is not a discrete one, but it includes a period of short-lived enhancement of ocular drift (or retinal-image slip) that likely alters visual responses in the retina and beyond. Second, microsaccades and their postmovement drift act to modulate visual sensitivity to full-field image motion, which is presumed to normally aid the visual and oculomotor systems to rapidly stabilize the world immediately after these rapid, flicklike eye movements.

\section{Discussion}

In this paper, we studied the characteristics of ocular drifts, and necessarily retinal-image slip, around the time of microsaccades. We found a period of enhanced retinal-image slip immediately after these movements, with no large change in such slip (either enhancement or reduction) before them. Moreover, we discovered that microsaccades also cause postmovement enhancement of early motion sensitivity, evidenced by a much more responsive ocular following response to full-field image motion when this motion starts immediately after microsaccades than when it starts without them. These results have implications on our understanding of the motor control of microsaccades, as well as their neural and perceptual consequences.

\section{Origins of postmicrosaccadic enhancement}

Our observation of postmicrosaccadic enhancement of ocular drift is similar to classic observations of enhanced drifts immediately after much larger saccades (Figs. $1 B, 3 B$, blue). Such classically observed postsaccadic drifts, or glissades (Weber and Daroff, 1972; Bahill et al., 1978), could either appear as backward eye position drifts opposite the saccade direction or as forward 
ones. Glissades were therefore initially described as corrective movements (Weber and Daroff, 1972), perhaps even being centrally programmed. However, the exact origin of postsaccadic drifts is not entirely known, with another possibility being related to small mismatches or variability in the oculomotor drive signals generating saccades (Easter, 1973; Bahill et al., 1978). Specifically, saccadic eye velocities are achieved at the level of brainstem oculomotor neurons by a "pulse-step" motor command: a strong initial pulse of action potentials that rapidly moves the eye at saccadic velocities, and a subsequent tonic discharge (step command) that maintains the eye at the saccade endpoint (Van Gisbergen et al., 1981). According to one view of postsaccadic drifts, if there is a small mismatch between the position that the eye lands on as a result of the "pulse" command and the "desired" position specified by the "step" command, then a slow eye movement might be expected to occur until the mismatch is corrected for (Easter, 1973; Bahill et al., 1978).

Whatever the origin of postsaccadic drifts, the remarkable observation of postmovement drifts even for the smallest microsaccades suggests that a similar mechanism must be at play for these tiny eye movements. This is consistent with observations that the brainstem control of saccades and microsaccades is shared (Van Gisbergen et al., 1981; Hafed et al., 2009; Hafed, 2011; Goffart et al., 2012; Hafed and Krauzlis, 2012). This would also be consistent with the hypothesis that microsaccades can cause a "gain change" in early motion processing as we saw in our data for the ocular following task (Figs. 6, 7), because larger saccades are also known to increase the gain of visuomotor transformations for ocular following (Kawano and Miles, 1986) (Fig. 7C) and smooth pursuit (Lisberger, 1998). Under normal circumstances, such a gain change is useful because it would allow the visual system to quickly stabilize gaze in the face of full-field image motion caused by postmicrosaccadic (and postsaccadic) drift.

\section{Are microsaccades triggered by reduced retinal-image slip?}

Regardless of what causes postmicrosaccadic (and postsaccadic) enhancement of ocular drift, our results also clarify an important question about what triggers microsaccades. Specifically, and using two different analysis approaches, we found no reductions in retinal-image slip, and for an extended period of up to $400-500$ $\mathrm{ms}$ before microsaccade onset (Figs. 3, 4). This is in direct contrast to an earlier result arguing that microsaccades are triggered by low retinal-image slip (Engbert and Mergenthaler, 2006; Engbert et al., 2011), but it is in agreement with later experiments that directly manipulated image visibility and found that microsaccades are not necessarily triggered by image fading (which would be the expected perceptual consequence of reduced retinal-image slip) (Poletti and Rucci, 2010).

We think that our results are different from those by (Engbert and Mergenthaler, 2006) because these authors used a videobased eye tracker (tracking pupil position in a video image), whereas we used scleral search coils in both monkeys for this sensitive analysis. Video-based eye trackers that measure pupil position are susceptible to small position artifacts due to changes in pupil diameter (Wyatt, 2010; Kimmel et al., 2012). In fact, when we directly re-analyzed monkey P's video-based eye tracker data for premicrosaccadic ocular drifts as in Figure $4 A$, we could indeed replicate the Engbert and Mergenthaler (2006) result (Fig. $8 B$ ), suggesting an interpretational ambiguity caused by the video-based data (Kimmel et al., 2012). This re-analysis then prompted us to collect even more fixation data from monkey $\mathrm{N}$, and now using simultaneous recording of the same eye with both
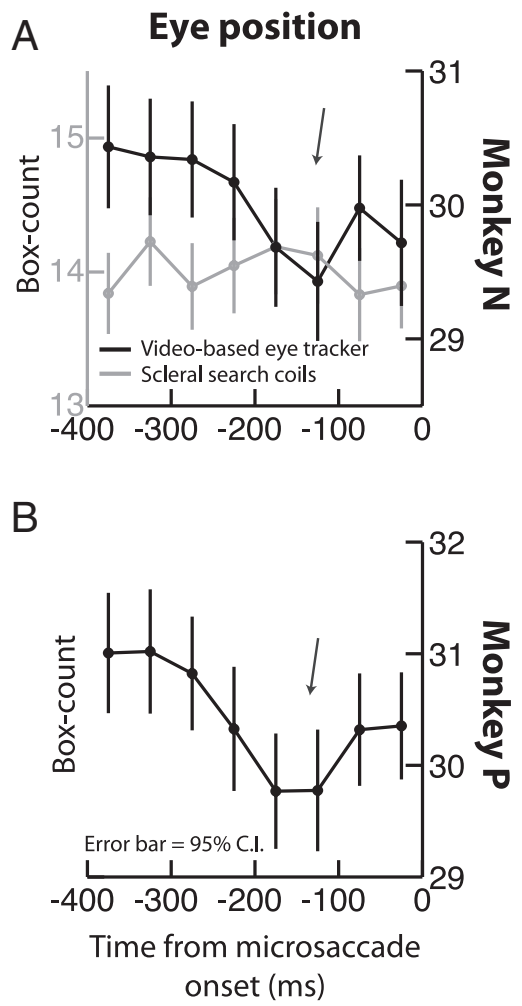

Figure 8. $\quad \boldsymbol{A}, \boldsymbol{B}$, Premicrosaccadic ocular drifts using different eye tracking techniques. We repeated the same box counting procedure of Engbert and Mergenthaler (2006) on both monkeys, but now using the video-based eye tracker (black curves). In monkey $N(\boldsymbol{A})$, we also measured the same eye simultaneously using scleral search coils (gray curve; note that the box counts for the coils are lower than those for the video-based eye tracker because coils are much less noisy). In both monkeys, the video-based data showed an apparently artifactual reduction in ocular drift $\sim 200$ ms before microsaccade onset, replicating Engbert and Mergenthaler (2006). This reduction was not present in simultaneously measured search coil data ( $\boldsymbol{A}$, gray) and in all our other analyses with coils (Figs. 1-5). This discrepancy likely reflects the limitation of video-based eye tracking for measuring slow ocular drifts (Kimmel et al., 2012). All conventions are similar to Figure $4 A$ and $C$.

scleral search coils and video-based eye tracking. Again, we could replicate the Engbert and Mergenthaler (2006) results in this second monkey, but only when we used the video-based tracker data (Fig. $8 \mathrm{~A}$, black); no reduction in premicrosaccadic retinal-image slip could be detected in the simultaneously recorded scleral search coil data (Fig. 8A, gray), consistent with our earlier results (Fig. 4). We therefore conclude that microsaccades are not necessarily triggered by reductions in retinal-image slip, consistent with other studies that directly tested the relationships between visual percepts of fading and microsaccades (Poletti and Rucci, 2010). This conclusion adds to the currently emerging picture of a decidedly varied and complex role of microsaccades in vision (Hafed et al., 2009, 2011; Rolfs, 2009; Hafed and Krauzlis, 2010; Ko et al., 2010; Kuang et al., 2012; McCamy et al., 2012; Hafed, 2013).

\section{Perceptual consequences of microsaccades}

Our results do indicate, however, that microsaccades indeed have perceptual consequences regardless of how they get triggered. In addition to the expected alteration of retinal images caused by microsaccades shifting the line of sight from one point in foveal space to another, our results indicate that retinal images are significantly modulated after microsaccades. This has implications on interpreting some neural and perceptual phenomena attrib- 
uted to microsaccades, because it indicates that the visual system experiences faster full-field image motion after microsaccades than otherwise. For example, there have been some conflicting observations about the neural consequences of microsaccades in the primary visual cortex (V1), with some studies emphasizing bursting after microsaccades (Martinez-Conde et al., 2000) and others reporting more varied responses (Kagan et al., 2008). Similar discrepancies have also been observed in extrastriate area V4 (Leopold and Logothetis, 1998; Bosman et al., 2009). Since the retinal-image motions associated with ocular drift can affect all of these early visual areas, our results may reconcile some of these discrepancies. For example, it could be the case that if responses in V1 are aligned on microsaccade end, some of the varied response patterns observed in (Kagan et al., 2008) can be explained by whether ocular drift moves images in preferred or nonpreferred neuronal directions.

Our results can also clarify the contribution of microsaccades to some perceptual phenomena. For example, the "rotating snakes" illusion is a motion illusion that has been hypothesized to be triggered by slow eye movements (Murakami et al., 2006). More recently, however, an additional contribution of microsaccades, and even blinks, was also demonstrated (Otero-Millan et al., 2012). Since our results show that enhanced slow eye movements follow microsaccades anyway, these results support a functional link between the two interpretations.

\section{Implications for active vision}

Finally, our results suggest that studies of active vision in the broader context, beyond just microsaccades, need to consider postsaccadic drifts in eye position and their potential contributions to altering neural and behavioral data. For example, neurons in area MST, which is implicated in mediating postsaccadic enhancement of ocular following (Takemura and Kawano, 2006; Ibbotson et al., 2007) (and presumably the postmicrosaccadic enhancement that we observed here), exhibit increases in spontaneous activity immediately after saccades, and particularly under well lit conditions (Ibbotson et al., 2008). These increases could reflect postsaccadic drifts that move the visual world over the receptive fields of these neurons. In fact, our observation of both postmicrosaccadic enhancement of ocular drift and postmicrosaccadic enhancement of ocular following responses suggest that under natural conditions, eye movements have the majority of the impact on neural processing in the visual brain. Specifically, the combination of postsaccadic enhancement as well as active extraretinal mechanisms associated with eye movements (including microsaccades), such as saccadic suppression (Diamond et al., 2000; Hafed and Krauzlis, 2010), saccadic compression (Ross et al., 1997; Hafed, 2013), and postsaccadic enhancement of motion sensitivity, suggests that the mere generation of a rapid eye movement can result in altered responses in the visual system for up to 250-300 ms around each movement. Given that eye movements can take place approximately three times a second, this suggests that perception is essentially under a constant influence of the retinal and extraretinal processes associated with moving the eye. If one also considers the constant perpetual drift that never ceases to occur between saccades, as well as the remarkable influence it might have on image statistics impinging on the retina (Kuang et al., 2012), one is compelled to consider the role of eye movements in any study associated with visual perception. Adopting such a view to research on "active" vision can be tremendously helpful for fully understanding visual function under natural conditions.

\section{References}

Bahill AT, Hsu FK, Stark L (1978) Glissadic overshoots are due to pulse width errors. Arch Neurol 35:138-142. CrossRef Medline

Bair W, O'Keefe LP (1998) The influence of fixational eye movements on the response of neurons in area MT of the macaque. Vis Neurosci 15:779_ 786. Medline

Barlow HB (1952) Eye movements during fixation. J Physiol 116:290-306. Medline

Bosman CA, Womelsdorf T, Desimone R, Fries P (2009) A microsaccadic rhythm modulates gamma-band synchronization and behavior. J Neurosci 29:9471-9480. CrossRef Medline

Boström KJ, Warzecha AK (2009) Ocular following response to sampled motion. Vision Res 49:1693-1701. CrossRef Medline

Brainard DH (1997) The Psychophysics Toolbox. Spat Vis 10:433-436. CrossRef Medline

Bremmer F, Kubischik M, Hoffmann KP, Krekelberg B (2009) Neural dynamics of saccadic suppression. J Neurosci 29:12374-12383. CrossRef Medline

Cherici C, Kuang X, Poletti M, Rucci M (2012) Precision of sustained fixation in trained and untrained observers. J Vis 12(6) pii:31.

Cloherty SL, Mustari MJ, Rosa MG, Ibbotson MR (2010) Effects of saccades on visual processing in primate MSTd. Vision Res 50:2683-2691. CrossRef Medline

Collewijn H, Kowler E (2008) The significance of microsaccades for vision and oculomotor control. J Vis 8(14):20 1-21. CrossRef Medline

Crowder NA, Price NS, Mustari MJ, Ibbotson MR (2009) Direction and contrast tuning of macaque MSTd neurons during saccades. J Neurophysiol 101:3100-3107. CrossRef Medline

Deubel H, Bridgeman B (1995) Fourth Purkinje image signals reveal eyelens deviations and retinal image distortions during saccades. Vision Res 35:529-538. CrossRef Medline

Diamond MR, Ross J, Morrone MC (2000) Extraretinal control of saccadic suppression. J Neurosci 20:3449-3455. Medline

Easter SS Jr (1973) A comment on the "glissade." Vision Res 13:881-882.

Engbert R, Mergenthaler K (2006) Microsaccades are triggered by low retinal image slip. Proc Natl Acad Sci U S A 103:7192-7197. CrossRef Medline

Engbert R, Mergenthaler K, Sinn P, Pikovsky A (2011) An integrated model of fixational eye movements and microsaccades. Proc Natl Acad Sci U S A 108:E765-E770. CrossRef Medline

Fuchs AF, Robinson DA (1966) A method for measuring horizontal and vertical eye movement chronically in the monkey. J Appl Physiol 21: 1068-1070. Medline

Gellman RS, Carl JR, Miles FA (1990) Short latency ocular-following responses in man. Vis Neurosci 5:107-122. CrossRef Medline

Goffart L, Hafed ZM, Krauzlis RJ (2012) Visual fixation as equilibrium: evidence from superior colliculus inactivation. J Neurosci 32:1062710636. CrossRef Medline

Hafed ZM (2011) Mechanisms for generating and compensating for the smallest possible saccades. Eur J Neurosci 33:2101-2113. CrossRef Medline

Hafed ZM (2013) Alteration of visual perception prior to microsaccades. Neuron 77:775-786. CrossRef

Hafed ZM, Clark JJ (2002) Microsaccades as an overt measure of covert attention shifts. Vision Res 42:2533-2545. CrossRef Medline

Hafed ZM, Krauzlis RJ (2010) Microsaccadic suppression of visual bursts in the primate superior colliculus. J Neurosci 30:9542-9547. Medline

Hafed ZM, Krauzlis RJ (2012) Similarity of superior colliculus involvement in microsaccade and saccade generation. J Neurophysiol 107:1904-1916. CrossRef Medline

Hafed ZM, Goffart L, Krauzlis RJ (2009) A neural mechanism for microsaccade generation in the primate superior colliculus. Science 323:940-943. CrossRef Medline

Hafed ZM, Lovejoy LP, Krauzlis RJ (2011) Modulation of microsaccades in monkey during a covert visual attention task. J Neurosci 31:15219-15230. CrossRef Medline

Hafed ZM, Lovejoy LP, Krauzlis RJ (2013) Superior colliculus inactivation alters the relationship between covert visual attention and microsaccades. Eur J Neurosci. Advance online publication. Retrieved Jan. 21, 2013. doi: 10.1111/ejn.12127. CrossRef

Herrington TM, Masse NY, Hachmeh KJ, Smith JE, Assad JA, Cook EP (2009) The effect of microsaccades on the correlation between neural 
activity and behavior in middle temporal, ventral intraparietal, and lateral intraparietal areas. J Neurosci 29:5793-5805. CrossRef Medline

Ibbotson MR, Price NS, Crowder NA, Ono S, Mustari MJ (2007) Enhanced motion sensitivity follows saccadic suppression in the superior temporal sulcus of the macaque cortex. Cereb Cortex 17:1129-1138. Medline

Ibbotson MR, Crowder NA, Cloherty SL, Price NS, Mustari MJ (2008) Saccadic modulation of neural responses: possible roles in saccadic suppression, enhancement, and time compression. J Neurosci 28:10952-10960. CrossRef Medline

Janabi-Sharifi F, Hayward V, Chen C-SJ (2000) Discrete-time adaptive windowing for velocity estimation. IEEE Trans Control Sys Tech 8:10031009. CrossRef

Judge SJ, Richmond BJ, Chu FC (1980) Implantation of magnetic search coils for measurement of eye position: an improved method. Vision Res 20:535-538. CrossRef Medline

Kagan I, Gur M, Snodderly DM (2008) Saccades and drifts differentially modulate neuronal activity in V1: effects of retinal image motion, position, and extraretinal influences. J Vis 8(14):19 1-25. CrossRef Medline

Kawano K, Miles FA (1986) Short-latency ocular following responses of monkey. II. Dependence on a prior saccadic eye movement. J Neurophysiol 56:1355-1380. Medline

Kimmel DL, Mammo D, Newsome WT (2012) Tracking the eye non-invasively: simultaneous comparison of the scleral search coil and optical tracking techniques in the macaque monkey. Front Behav Neurosci 6:49. Medline

Ko HK, Poletti M, Rucci M (2010) Microsaccades precisely relocate gaze in a high visual acuity task. Nat Neurosci 13:1549-1553. CrossRef Medline

Krauzlis RJ, Miles FA (1996) Release of fixation for pursuit and saccades in humans: evidence for shared inputs acting on different neural substrates. J Neurophysiol 76:2822-2833. Medline

Kuang X, Poletti M, Victor JD, Rucci M (2012) Temporal encoding of spatial information during active visual fixation. Curr Biol 22:510-514. CrossRef Medline

Leopold DA, Logothetis NK (1998) Microsaccades differentially modulate neural activity in the striate and extrastriate visual cortex. Exp Brain Res 123:341-345. CrossRef Medline

Lisberger SG (1998) Postsaccadic enhancement of initiation of smooth pursuit eye movements in monkeys. J Neurophysiol 79:1918-1930. Medline

Lovejoy LP, Krauzlis RJ (2010) Inactivation of primate superior colliculus impairs covert selection of signals for perceptual judgments. Nat Neurosci 13:261-266. CrossRef Medline

Martinez-Conde S, Macknik SL, Hubel DH (2000) Microsaccadic eye movements and firing of single cells in the striate cortex of macaque monkeys. Nat Neurosci 3:251-258. CrossRef Medline

Martinez-Conde S, Macknik SL, Hubel DH (2002) The function of bursts of spikes during visual fixation in the awake primate lateral geniculate nucleus and primary visual cortex. Proc Natl Acad Sci U S A 99:13920-13925. CrossRef Medline

Masson GS, Perrinet LU (2012) The behavioral receptive field underlying motion integration for primate tracking eye movements. Neurosci Biobehav Rev 36:1-25. CrossRef Medline

Matsuura K, Miura K, Taki M, Tabata H, Inaba N, Kawano K, Miles FA
(2008) Ocular following responses of monkeys to the competing motions of two sinusoidal gratings. Neurosci Res 61:56-69. CrossRef Medline

McCamy MB, Otero-Millan J, Macknik SL, Yang Y, Troncoso XG, Baer SM, Crook SM, Martinez-Conde S (2012) Microsaccadic efficacy and contribution to foveal and peripheral vision. J Neurosci 32:9194-9204. CrossRef Medline

Miles FA, Kawano K, Optican LM (1986) Short-latency ocular following responses of monkey. I. Dependence on temporospatial properties of visual input. J Neurophysiol 56:1321-1354. Medline

Murakami I, Kitaoka A, Ashida H (2006) A positive correlation between fixation instability and the strength of illusory motion in a static display. Vision Res 46:2421-2431. CrossRef Medline

Otero-Millan J, Macknik SL, Martinez-Conde S (2012) Microsaccades and blinks trigger illusory rotation in the "rotating snakes" illusion. J Neurosci 32:6043-6051. CrossRef Medline

Pelli DG (1997) The VideoToolbox software for visual psychophysics: transforming numbers into movies. Spat Vis 10:437-442. CrossRef Medline

Poletti M, Rucci M (2010) Eye movements under various conditions of image fading. J Vis 10(3):6 1-18. CrossRef Medline

Quaia C, Sheliga BM, Fitzgibbon EJ, Optican LM (2012) Ocular following in humans: spatial properties. J Vis 12(4) pii:13. CrossRef Medline

Rajkai C, Lakatos P, Chen CM, Pincze Z, Karmos G, Schroeder CE (2008) Transient cortical excitation at the onset of visual fixation. Cereb Cortex 18:200-209. Medline

Reppas JB, Usrey WM, Reid RC (2002) Saccadic eye movements modulate visual responses in the lateral geniculate nucleus. Neuron 35:961-974. CrossRef Medline

Rolfs M (2009) Microsaccades: small steps on a long way. Vision Res 49: 2415-2441. CrossRef Medline

Ross J, Morrone MC, Burr DC (1997) Compression of visual space before saccades. Nature 386:598-601. CrossRef Medline

Sheliga BM, Fitzgibbon EJ, Miles FA (2008) Spatial summation properties of the human ocular following response (OFR): evidence for nonlinearities due to local and global inhibitory interactions. Vision Res 48:1758-1776. CrossRef Medline

Takemura A, Kawano K (2006) Neuronal responses in MST reflect the postsaccadic enhancement of short-latency ocular following responses. Exp Brain Res 173:174-179. CrossRef Medline

Van Gisbergen JA, Robinson DA, Gielen S (1981) A quantitative analysis of generation of saccadic eye movements by burst neurons. J Neurophysiol 45:417-442. Medline

Verheijen FJ (1961) A simple after image method demonstrating the involuntary multidirectional eye movements during fixation. Opt Acta 8:309_ 311. CrossRef Medline

Weber RB, Daroff RB (1972) Corrective movements following refixation saccades: type and control system analysis. Vision Res 12:467-475. CrossRef Medline

Wyatt HJ (2010) The human pupil and the use of video-based eyetrackers. Vision Res 50:1982-1988. CrossRef Medline 